\title{
Escoamento Atmosférico sobre uma Colina Isolada Revelado a Partir de Dados de um Veículo Aéreo não Tripulado (VANT)
}

\author{
Arcilan T. Assireu ${ }^{1}$ (D) José C. Mendonça ${ }^{2}$ (D), Ramon M. Freitas ${ }^{3}$ (D), Robson B. Passos ${ }^{1}$ (D), \\ Cláudio C. Pellegrini ${ }^{4}$ (D), Felipe M. Pimenta ${ }^{5}$ (i) \\ ${ }^{1}$ Universidade Federal de Itajubá, Itajubá, MG, Brasil. \\ ${ }^{2}$ Universidade Estadual do Norte Fluminense, Campos dos Goytacazes, RJ, Brasil. \\ ${ }^{3}$ Camargo \& Schubert Engenharia do Vento, Curitiba, PR, Brasil. \\ ${ }^{4}$ Universidade Federal de São João Del, São João del Rei, MG, Brasil. \\ ${ }^{5}$ Universidade Federal de Santa Catarina, Florianópolis, SC, Brasil.
}

Recebido em: 25 de Setembro de 2017 - Aceito em: 10 de Dezembro de 2017

\begin{abstract}
Resumo
Este artigo é uma contribuição do Projeto “uso de VANT para prospecção eólica em sistemas aquáticos”, que consistiu num esforço multi-instrumental e multi-institucional para estudar a camada limite atmosférica influenciada por extensos corpos d'água e pelas feições do entorno como colinas, rampas e mudanças abruptas de rugosidade. O trabalho apresenta o resultado de um experimento específico, realizado em novembro de 2016, que visou estudar a camada limite atmosférica (CLA) influenciada por colinas. Durante os experimentos foram levantados perfis verticais do vento a partir de um VANT, utilizado pela primeira vez no Brasil, para esta finalidade. O escoamento do vento, no topo da colina, apresentou forte intensificação a $20 \mathrm{~m}$ de altura. Os resultados foram comparados com um modelo analítico baseado na teoria linear aplicado a um terreno real.
\end{abstract}

Palavras-chave: escoamento em colinas, aceleração do vento, energia eólica.

\section{Flow over an isolated hill revealed by multirotor aircraft data}

\begin{abstract}
This paper represents a contribution of the Project "unmanned aerial vehicles to wind assessment in aquatic systems", a multi-institutional and multi-instrumented experiment carried out to measure, in detail, the characteristics of winds over a large tropical aquatic system and how the flow is modulated by hill, ramp and abrupt roughness changes. It presents results from experiments conducted during november 2016 over a typical hill site that is adequate for installation of wind turbines. During the experiments, both hilltop and upstream wind profile data were obtained using an unmanned rotorcraft, commonly known as a hexacopter. The flow speed at $20 \mathrm{~m}$ over the top of hill was found to be increased by a factor of 1.8. The results were compared with an analytical model which makes a careful application of linear theory to real terrain.
\end{abstract}

Keywords: hill flow, flow speed-up, wind energy.

\section{Introdução}

O escoamento atmosférico influenciado por colinas é um tema de pesquisa motivado por diferentes aplicações práticas como a dispersão de poluentes (Manikantan e Pruthviraj, 2010), o aproveitamento eólico (Mattuella et al., 2016) e, mais recentemente, a propagação de queimadas em florestas (Forthofer et al., 2014). Um dos aspectos muito bem documentado na literatura é a aceleração do escoamento no topo das colinas. Este fenômeno será o foco deste trabalho, devido às suas implicações práticas para a energia eólica, uma vez que a densidade de potência é proporcional ao cubo da velocidade do vento.

Autor de correspondência: Arcilan T. Assireu, arcilan@unifei.edu.br. 
Estudos sobre o efeito das colinas para o escoamento do vento são baseados em modelos físicos em escala reduzida ensaiados em túneis de vento (e.g., Vladut et al., 2015), modelagem numérica (e.g., Chaudhari et al., 2014; Schmidt e Stoevesandt, 2014) e, ainda, em escassos experimentos realizados especificamente para levantar dados do escoamento em colinas (e.g., Berg et al., 2011). Em relação aos experimentos em túneis de vento, Athanassiadou e Castro (2001) examinaram o escoamento em condições neutras sobre uma série de colinas bidimensionais e compararam com o escoamento em áreas sem a presença de colinas. Ayotte e Hughes (2004) estudaram o escoamento bidimensional turbulento, em condição de estratificação neutra, sobre regiões com declividades variadas e colinas isoladas e múltiplas, com o objetivo da melhor compreensão da transição de casos onde a teoria linear é válida para o caso onde isto não ocorre. Cao e Tanura $(2006$; 2007) conduziram medidas relativas ao impacto das mudanças de rugosidade para o escoamento bidimensional em rampas e em colinas. No caso das colinas, ênfases foram dadas para o efeito da mudança de rugosidade sobre o escoamento médio e turbulento. $\mathrm{O}$ efeito da aceleração do escoamento e a influência da direção do vento para a aceleração em escoamentos tridimensionais sobre colinas elípticas e circulares foram examinados por Lubitz e White (2007) e Liu et al. (2016). Análises para o escoamento influenciado por colinas, baseadas em modelos numéricos são bem documentadas. Argain et al. (2009) apresentam uma formulação para a velocidade de fricção influenciada por estas feições. Lee et al. (2003) estudaram o efeito da distância entre duas colinas para as características do escoamento e indicaram que uma colina vizinha pode ter um efeito significativo sobre o padrão do escoamento para distâncias de até 6 vezes a largura característica da colina. Bitsuamlak et al. (2004) fizeram uma revisão de estudos sobre modelagem numérica aplicados a diferentes tipos de topografia, incluindo colinas, e concluíram que a boa concordância com resultados observados em túnel de vento torna promissora a aplicação dos modelos numéricos em estudos do escoamento atmosférico influenciado por colinas. Paiva et al. (2004), fazendo uso do modelo regional atmosférico brasileiro (BRAMS), compararam os resultados deste modelo com os do experimento do projeto Askervein e concluíram que os perfis gerados são fortemente dependentes da velocidade de fase da onda de gravidade interna especificada no modelo. Pellegrini e Bodstein (2005) propuseram uma lei logarítmica modificada para descrever a camada limite planetária (CLP) sobre uma colina bidimensional em condições atmosféricas neutras. Os autores chegaram a uma formulação que depende de um parâmetro que representa a influência da forma da colina para o escoamento.

As escassas campanhas de campo que visaram gerar medidas sobre o escoamento influenciado por colinas são baseadas no histórico Projeto Colina Askervein, que ocorreu entre 1982 e 1983 e um mais recente (2007 e 2008), realizado na Dinamarca, conhecido como campanha Bolund (Berg et al., 2011).

O projeto Colina Askervein foi um extensivo estudo de escoamento sobre colinas conduzido em 1982, na forma de testes preliminares e, em 1983 como estudos completos. O principal foco foi o estudo das características espaciais do escoamento médio e turbulento sobre uma colina típica com características que permitem o aproveitamento eólico, localizada em uma cadeia de ilhas na costa oeste da Escócia. Este experimento foi usado extensivamente para estudos de casos com modelagem numérica como em Castro et al. (2003), Paiva et al. (2004), Pellegrini e Bodstein (2005), Lopes et al. (2007), dentre outros. O outro experimento importante foi realizado na colina Bolund que apresenta $12 \mathrm{~m}$ de altura e $130 \mathrm{~m}$ de extensão, localizada na Dinamarca. Os principais resultados deste projeto podem ser encontrados em Bechman et al. $(2009 ; 2011)$.

Enquanto o uso de VANTs de asa fixa em várias aplicações é bastante documentado (e.g., Dias et al., 2012), os VANTs de asa móvel (quadricópteros, hexacópteros, etc.), aplicados a estudos geofísicos é bastante recente. Estes VANTs vêm sendo utilizados para estudos sobre o impacto da poeira para o albedo da neve (Di Mauro et al., 2015), emissão de metano (Brownlow et al., 2016), modelagem da paisagem estatigráfica (Nieminski et $a l ., 2017$ ), estudos sobre qualidade de água (Ore et al, 2015), mapeamento da drenagem glacial (Rippin et al., 2015), mapeamento de corpos d'água intermitentes (Spence e Mengistu, 2016) e aplicações em estudos relacionados à ecologia aquática (Vivoni et al., 2014). O objetivo deste trabalho é descrever o fenômeno de aceleração dos ventos sobre colinas, a partir de dados medidos por um VANT no reservatório hidrelétrico de Furnas, MG. O perfil vertical observado foi analisado e comparado com modelos analíticos desenvolvidos por Pellegrini e Bodstein (2005). Este estudo soma-se a outros, como Medeiros et al. (2013) que fizeram uso de observações de campo para a melhor descrição da CLP influenciada por terrenos complexos. Até onde se sabe, estas são as primeiras medidas do perfil vertical do vento realizadas através de VANTs no entorno grandes reservatórios no Brasil.

\section{Material e Métodos}

Este artigo traz os resultados das medidas de campo realizadas por dois projetos científicos: "Aproveitamento eólico em reservatórios hidrelétricos" e "Uso de VANT para prospecção eólica em sistemas aquáticos", ambos apoiados pela Fundação de Amparo à Pesquisa do Estado de Minas Gerais (FAPEMIG). Estes projetos, que envolveram cooperação entre várias instituições como Universidade Federal de Itajubá (UNIFEI), Universidade Federal de Santa Catarina (UFSC), Universidade Estadual 
do Norte Fluminense (UENF), Universidade Federal de São João Del Rei (UFSJ) e Instituto Nacional de Pesquisas Espaciais (INPE), representam um esforço para estudar a camada limite atmosférica em extensos sistemas aquáticos brasileiros. Maiores detalhes sobre os projetos, instrumentação utilizada e desenho amostral é dado em Assireu et al. (2018).

A região de estudo compreende o reservatório de Furnas, situado no interior do estado de Minas Gerais (Fig. 1a). A barragem da Usina Hidrelétrica de Furnas está localizada no curso médio do rio Grande, no trecho denominado "Corredeiras das Furnas", entre os municípios de São José da Barra e São João Batista do Glória, em Minas Gerais. A represa cobre uma superfície de $1440 \mathrm{~km}^{2}$ com a cota máxima de $762 \mathrm{~m}$ em relação ao nível do mar e abrange 34 municípios de Minas Gerais. O braço do reservatório em estudo, correspondente ao município de Guapé (MG), que vem sendo alvo de estudos que visam ou visaram estimativas do potencial eólico da região. $\mathrm{O}$ experimento foi realizado entre os dias 21 e 25 de novembro de $2016 \mathrm{em}$ diferentes setores do reservatório. No dia 22 de novembro as 09:00h (HL) foi amostrada uma colina com condições morfométricas e de acesso que poderiam receber um parque eólico (Fig. 1). Um mastro anemométrico de $10 \mathrm{~m}$ já havia sido instalado no topo da colina (Fig. 1b). O perfil vertical com o VANT, até $100 \mathrm{~m}$, consistiu de medidas a partir de $3 \mathrm{~m} \mathrm{e}$, a partir daí, a cada $5 \mathrm{~m}$. Em cada um dos níveis o VANT realizou medições de 1 minuto de duração. Como a taxa amostral foi de $10 \mathrm{~s}$, cada medida de vento, para cada altura, representa a média de 6 valores.

O uso de um hexacóptero, ao invés dos mais comuns quadricópteros, é devido a maior estabilidade do primeiro em relação ao último. Os seis motores trabalham em sincronia de forma a compensar eventuais perturbações causadas por turbulência. O controle de altitude é feito por um barômetro e os motores operam de forma a manter a pressão e, consequentemente, a altitude constante. $\mathrm{O}$ anemômetro utilizado e hospedado como carga útil no VANT
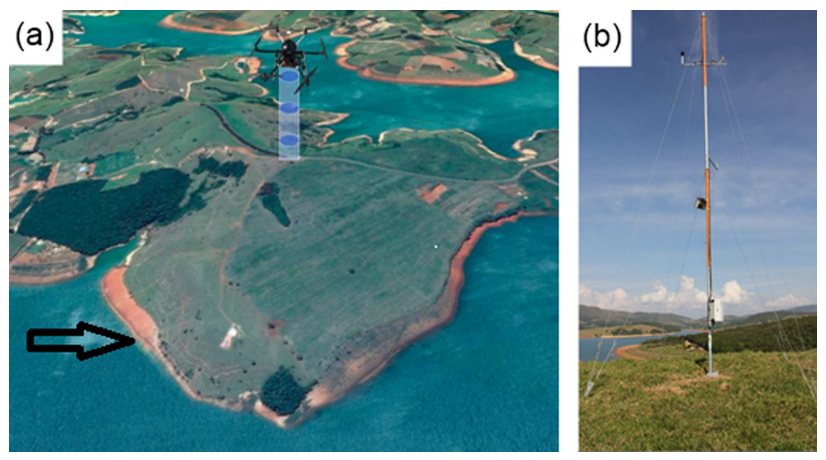

Figura 1 - Colina situada no braço do reservatório de Furnas onde foram levantados os perfis com o VANT (a) (seta indica direção do vento prevalente). Mastro anemométrico de $10 \mathrm{~m}$ situado no topo da colina (b). foi um modelo do tipo copo da marca Lambrecht. Um datalogger modelo CR1000 da Campbell, instalado próximo às baterias do VANT, foi utilizado para o armazenamento dos dados medidos (maiores detalhes vide Assireu et al., 2018). $\mathrm{O}$ anemômetro foi preso pelo cabo elétrico de conexão a $4 \mathrm{~m}$ abaixo do VANT. Esta distância foi empiricamente obtida como a distância a partir da qual a esteira dos motores não influencia as medidas do anemômetro. Isto evitou problemas como aqueles relatados por Palomaki et al. (2017), que reportaram medidas influenciadas pela esteira do VANT. Como o anemômetro foi fixado ao VANT pelo próprio cabo de comunicação com o dataloger, o esperado movimento pendular ocorre apenas imediatamente a decolagem, e estabiliza após, sem comprometer as medidas. A Tabela 1 traz a especificação do VANT e do anemômetro utilizado neste trabalho.

\section{Descrição da colina e modelo conceitual}

A Fig. 2 traz as principais características morfométricas da colina estudada aqui, obtidas a partir do modelo digital de terreno. Os principais parâmetros geométricos para descrição da colina são sua altura $(\mathrm{h} \sim 50 \mathrm{~m})$, o raio de curvatura superficial $\left(\mathrm{R}_{\mathrm{h}} \sim 90 \mathrm{~m}\right)$ e sua escala de comprimento horizontal $\left(\mathrm{L}_{\mathrm{h}} \sim 150 \mathrm{~m}\right)$, na direção do vento predominante. $\mathrm{O}$ comprimento de escala $\mathrm{L}_{\mathrm{h}}$ é definido como a distância horizontal da face da colina até o ponto correspondente a metade da altura da colina (h/2) (Fig. 2). A linha de fluxo é suposta suficientemente próxima à superfície o que é equivalente a assumir que o escoamento é paralelo à curvatura, conforme demonstrado experimentalmente por Salmon et al. (1988). O estado da arte do desenvolvimento da teoria empregada para análises do escoamento influenciado por colinas está muito bem documentado em Pellegrini e Bodstein (2005) e Bitsuamlak (2004) e maiores detalhes podem ser encontrados nestes trabalhos.

No perfil do vento à esquerda da Fig. 2, o termo $\mathrm{u}_{0}(\mathrm{z})$ corresponde à região a barlavento não influenciada pela colina e será denominado daqui em diante como perfil

Tabela 1 - Especificações do VANT e anemômetro utilizados neste trabalho.

\begin{tabular}{cc}
\hline \multicolumn{2}{c}{ Especificações do VANT e anemômetro utilizados } \\
\hline Tipo & Hexacóptero X700 \\
Dimensões & $1057 \times 723 \times 462 \mathrm{~mm}$ \\
Distância entre os rotores & $361.5 \mathrm{~mm}$ \\
Peso & $1750 \mathrm{~g}$ (com bateria) \\
Autonomia & $20 \mathrm{a} 25$ minutos \\
Tempo de recarga da bateria & 120 minutos \\
Anemômetro & Copo (marca Lambrecht) \\
Peso & $0,9 \mathrm{~kg}^{-1}$ \\
Intervalo & 0,7 a $50,0 \mathrm{~m} \mathrm{~s}^{-1}$ \\
Resolução & $0,26 \mathrm{~m} \mathrm{~s}^{-1}$ \\
\hline
\end{tabular}




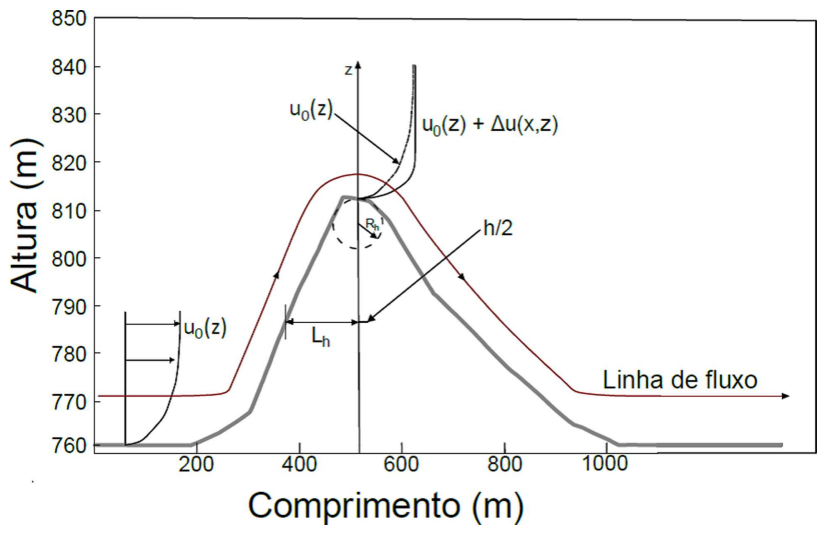

Figura 2 - Morfometria da colina analisada e os respectivos parâmetros geométricos.

de referência (PR). No topo da colina, o perfil médio é dado por $u(\mathrm{x}, \mathrm{z})=\mathrm{u}_{0}(\mathrm{z})+\Delta u(\mathrm{x}, \mathrm{z})$, onde a aceleração $\Delta u$ é causada por variações da topografia. Esta aceleração é positiva no topo da colina (TC) devido à condição de continuidade do escoamento do fluído. Uma abordagem formal para este efeito pode ser encontrada em Kilpatrick (2016). A razão entre a aceleração $\Delta u(\mathrm{x}, \mathrm{z}) \mathrm{e}$ a velocidade de referência $\mathrm{u}_{0}(\mathrm{z})$, define a aceleração relativa $\Delta S(\mathrm{x}, \mathrm{z})$ :

$$
\Delta S(\mathrm{x}, \mathrm{z})=\frac{\Delta u(\mathrm{x}, \mathrm{z})}{u_{0}(\mathrm{x}, \mathrm{z})}=\frac{u(\mathrm{x}, \mathrm{z})}{u_{0}(\mathrm{x}, \mathrm{z})}-1
$$

A altura $(l)$ onde ocorre o máximo aumento relativo da velocidade $\left(\Delta S_{\text {máx }}\right)$ é de especial interesse, pois fornece subsídios para definições como alturas de chaminés, aerogeradores, máximo arrasto sobre estruturas, dentre outros aspectos relacionados ao escoamento sobre colinas. Algumas expressões utilizadas para a estimativa de $\Delta S$ e $l$ podem ser encontradas em Walmsley e Taylor (1996) e Poggi et al. (2008), que fizeram uma boa revisão sobre este assunto. Este trabalho focará em duas expressões para estimativa de $l$ e para $\Delta S$. Uma, proposta por Finnigan (1992) e que leva em conta aspectos geométricos da colina anteriormente definidos:

$$
\frac{L_{h}}{R_{h}} \ln (l)=C_{1} k^{2}
$$

onde a constante $C_{1}$ é determinada a partir de comparação com dados observados. A outra equação a ser utilizada foi proposta por Pellegrini e Bodstein (2000):

$$
l \ln (l)=2,41 k^{2} L_{h}
$$

neste caso $l$ é obtido a partir de iterações que assegurem convergência entre os termos do lado esquerdo e direito de (3).

Hunt (1980) propõe a seguinte expressão para uma colina 2D:

$$
\Delta S \approx 2 \frac{h}{L_{h}}
$$

Enquanto Taylor e Lee (1984) sugerem:

$$
\Delta S \approx 1,6 \frac{h}{L_{h}}
$$

Para inferências sobre a condição atmosférica durante os experimentos foram utilizados os dois formalismos mais comumente empregados para esta finalidade: Número de Richardson Bulk $\left(R_{b}\right)$ e Gradiente $\left(R_{i}\right)$.

\subsection{Modelo a partir do Número de Richardson Bulk}

Neste método, as medições da temperatura do ar e da água são usadas em conjunto com a intensidade do vento a uma determinada altura (Shimada et al., 2014):

$$
R_{b}=-\frac{g}{\theta_{v}} \frac{z \Delta \theta_{v}}{U^{2}}
$$

em que $U$ é a velocidade do vento, $\theta_{v}$ é a média da temperatura potencial virtual entre os dois níveis na atmosfera, e $\Delta \theta_{v}$ indica a variação da temperatura potencial virtual entre o ar e a água $\left(\theta_{v(\text { (ar) }}-\theta_{v \text { (água) })}\right)$. O parâmetro de estabilidade por meio do número de Richardson Bulk tem grande aplicação em regiões de transição terra-água, pois leva em consideração não só a temperatura do ar, mas também a temperatura superficial da água.

Em reservatórios tropicais, a pequena variação da temperatura da água, causada por sua elevada capacidade térmica, faz com que a mesma se aqueça mais lentamente em relação à superfície terrestre adjacente. Como consequência, durante o dia a atmosfera próxima à superfície da água se apresenta mais fria em relação à níveis mais elevados da atmosfera, favorecendo condições de estabilidade e, durante a noite, esta região superficial se mantém mais aquecida, o que favorece a instabilidade (Assireu et al., 2011).

A turbulência na CLP depende das condições de estabilidade atmosférica conduzidas pelo aquecimento ou resfriamento da superfície terrestre e das características da interação entre o campo de velocidade e a superfície. Um indicador dessas condições de estabilidade é o número de Richardson Gradiente (Johnson e Kelley, 2000).

Neste método, temperatura potencial virtual e velocidade do vento em duas alturas diferentes são usadas para estimar o número de Richardson Gradiente (Stull, 1991):

$$
R i=\frac{g}{\theta_{\mathrm{v}}} \frac{\left(\frac{\Delta \theta_{\mathrm{v}}}{\Delta z}\right)}{\left(\frac{\Delta u}{\Delta z}\right)^{2}}
$$

$\mathrm{Na}$ Eq. (7), $g$ corresponde à aceleração da gravidade $\left(9,8 \mathrm{~m} \mathrm{~s}^{-2}\right), \Delta u / \Delta z(1 / \mathrm{s})$ é o cisalhamento vertical do vento, $\theta_{v}(\mathrm{~K})$ é a média da temperatura potencial virtual entre os 
dois níveis e o termo $\Delta \theta / \Delta z$ é o gradiente vertical da temperatura potencial virtual.

Newman e Klein (2014) utilizaram parte da subdivisão criada por eles, considerando $R i<-0,2$ como atmosfera fortemente instável, $-0,2 \leq R i<-0,1$, instável, $-0,1 \leq$ $R i<0,1$, atmosfera neutra, $0,1 \leq R i<0,25$, estável e, valores superiores a 0,25 , fortemente estável.

As rotinas utilizadas em todos os cálculos acima são descritas em Johnson e Kelley (2000) e são disponíveis em (https://www.nrel.gov/wind/data-tools.html).

\section{Resultados e Discussão}

Como antecipado na literatura (e.g., Paiva et al., 2004; Pellegrini e Bodstein, 2005), o escoamento no topo de colinas tende a ser intensificado devido a conservação de massa associado ao adensamento das linhas de fluxo ao contornarem a colina. O perfil de vento obtido pelo VANT (Fig. 3a) confirmam experimentalmente este fato e apontam para um aumento de até $90 \%$, próximo a $20 \mathrm{~m}$ de altura, quando comparado ao PR (Fig. 3b). As medidas com o VANT apresentam acurácia de $0,5 \mathrm{~m} \mathrm{~s}^{-1}$ (Assireu et al., 2018), portanto, as diferenças observadas entre o perfil de referência (barlavento da colina) e o perfil no topo da colina, são significativamente diferentes, chegando a $4 \mathrm{~m}$ $\mathrm{s}^{-1}$ (Fig. 3a). Os perfis apresentados na Fig. 3 são muito parecidos aos obtidos por Paiva et al. (2004) (vide Fig. 7) via RAMS e corroborado com os dados experimentais para a colina de Askervein. Conforme pode ser visto, o perfil verificado na colina não segue o clássico crescimento logarítmico da velocidade com a altura esperado para terrenos planos. Assim, a existência de velocidades máximas em torno de $20 \mathrm{~m}$ indica que turbinas muito altas não necessariamente deverão reverter em maiores aproveitamentos energéticos. Aerogeradores de menor altura

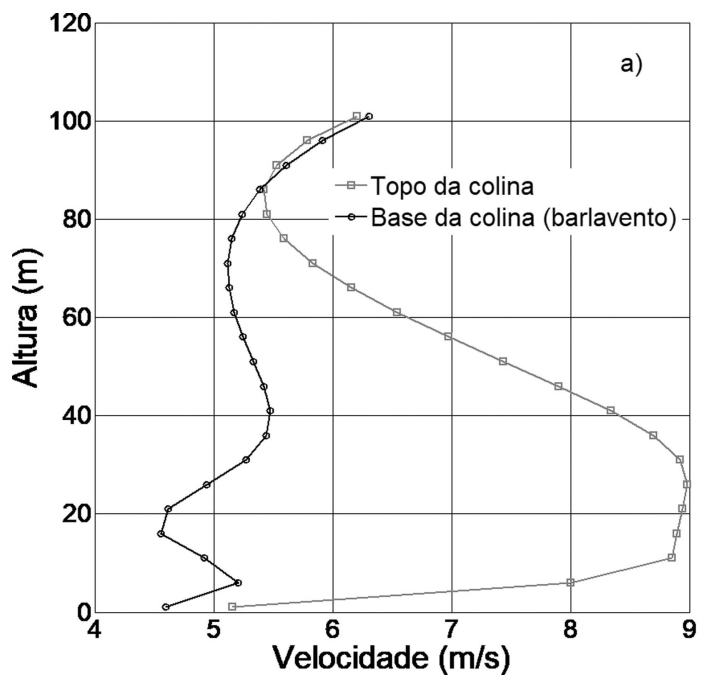

(porte) possivelmente alcancem maiores fatores de capacidade, também reduzindo custos e dificuldades logísticas.

A inclinação média da colina $(\Theta)$ do lado barlavento pode ser estimado por $50 \mathrm{~m} / 250 \mathrm{~m}$, o que resulta em $\Theta=0,2$. Se assumido o $\Theta_{c}$ (ângulo crítico) de 0,3 (Wood, 1995) como sendo o limiar para a aplicação da teoria linear (fluxos sobre a colina representados por linhas de corrente), percebe-se que a colina em estudo se enquadra nesta suposição. Assim, as eqs. (2) a (5) podem ser aplicadas. Para efeito de comparação, os valores de $\Theta$ para a colina de Askervein são indicados na literatura como variando entre 0,35 e 0,53 . Portanto, a colina neste estudo é mais suave do que Askervein.

A aplicação dos parâmetros da colina em estudo na Eq. (2) permitiu a obtenção da constante $\mathrm{C}_{l}$ tendo indicado o valor de 0,79 . Porém, a constante não é universal e dependente das condições de estabilidade da atmosfera. Pellegrini e Bodstein (2005) apontaram variação de cerca de $400 \%$ nesta constante durante o experimento de Askervein. Assim, a formulação proposta por Pellegrini e Bodstein (2005) (Eq. 3) parece mais adequada para fins práticos e foi aqui resolvida iterativamente. $\mathrm{O}$ valor teórico de $l$ que resultou em melhor convergência foi 19,5 m. Esta referese à altura teórica onde ocorre o máximo aumento relativo da velocidade. É surpreendente a aderência do valor previsto com aquele observado pelo VANT (Fig. 3b). O modelo proposto por Pellegrini e Bodstein (2005) deveria, a rigor, apresentar melhor desempenho em condições de atmosfera neutra e para escoamentos bi-dimensionais, ou seja, pouco influenciados por convergência lateral.

No momento que os perfis sobre a colina foram levantados não se dispunha de dados que permitissem avaliar a condição atmosférica quanto à estabilidade. Porém, tendo em vista inferir sobre a condição atmosférica no momento das medidas, será analisada a evolução

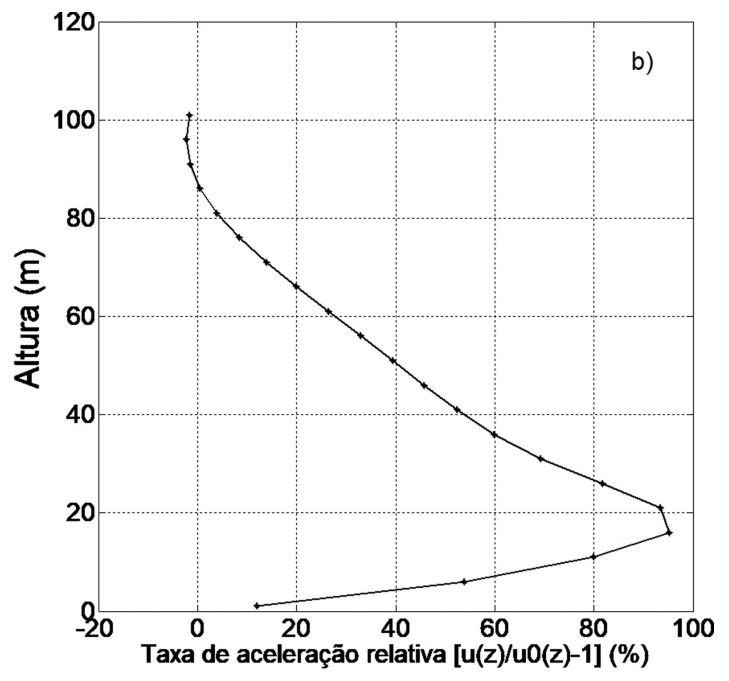

Figura 3 - (a) Perfis de velocidade no topo da colina (TC) e na base da colina a barlavento (perfil de referência - PR) (b) Perfil do aumento relativo da velocidade. 
diurna típica do Número de Richardson (Ri) para a área de estudo. Os dados a serem analisados são provenientes de um estudo realizado na região de estudo entre os dias 19 e 22 de setembro de 2016, quando foi utilizado um LIDAR montado sobre uma bóia flutuante para medidas do vento até $200 \mathrm{~m}$ e balões cativos para o levantamento do perfil de temperatura e umidade. As medidas de temperatura e umidade a partir do balão, realizadas a cada minuto, foram baseados em sensores muito compactos e leves, chamados Ibutton (Modelo DS1923) com resolução de $0,5{ }^{\circ} \mathrm{C}$ e intervalos de medidas entre -20 a $85^{\circ} \mathrm{C}$. Maiores detalhes sobre este campo vide Assireu et al. (2018). A Fig. 4 traz a série temporal, com média de $10 \mathrm{~min}$, da intensidade e direção de vento, temperatura (ar e água) e do Número de Richardson. Para a direção, ventos de norte são definidos com zero grau e aumentam seguindo a rotação horária. A intensidade do vento, no início (16:30 h - 19:00 h), mostra-se bastante variável, com valores flutuando entre 2 e $6 \mathrm{~m} \mathrm{~s}^{-1}$ e moderada variação vertical (cisalhamento), e com direção predominante de NW. A partir daí, até

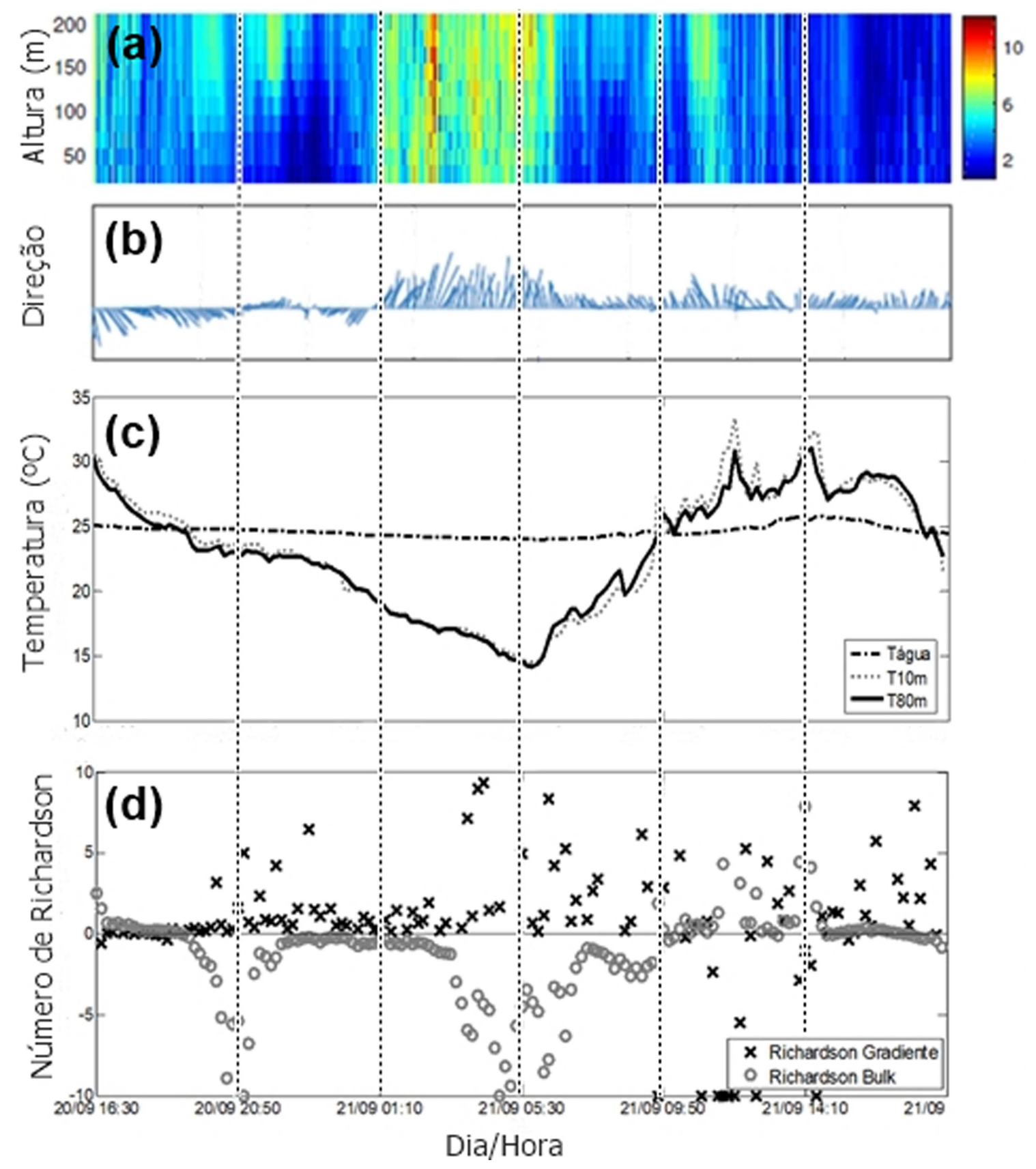

Figura 4 - Evolução diurna típica da intensidade e direção do vento (a,b), temperatura do ar e da água(c) e do Número de Richardson (d), para a área de estudo. 
aproximadamente 22:00 h, há um giro dos ventos coincidindo com expressivo aumento do cisalhamento, com ventos desde $2 \mathrm{~m} \mathrm{~s}^{-1}$ em superfície até $8 \mathrm{~m} \mathrm{~s}^{-1}$ a partir de $150 \mathrm{~m}$. Na sequência, observa-se uma inversão na direção, quando o vento passa a incidir a partir de $\mathrm{S}$ com valores mais intensos desde a superfície até $200 \mathrm{~m}$. Este vento, que ocorreu em 21/09 entre 00:00 e 07:00h, coincide com expressiva área de água sobre a qual o vento escoa (fetch) antes de atingir a plataforma de medida. $\mathrm{O}$ fetch é conhecido como área favorável a intensificação do vento (Holman et al., 2017).

A Fig. 4c traz a evolução diurna da temperatura da superfície da água, e da temperatura do ar a 10 e $80 \mathrm{~m}$. Estes dados foram utilizados nas estimativas do número de Richardson Bulk $\left(\mathrm{Ri}_{\mathrm{B}}\right)$ e Richardson Gradiente $\left(\mathrm{Ri}_{\mathrm{G}}\right)$ seguindo as formulações de Verburg e Antenucci (2010) e Johnson e Kelley (2000).

As condições atmosféricas neste campo e no período de coleta com o VANT eram muito similares, com poucas nuvens (Fig. 5a) e onde a evolução diurna da forçante radiativa modulou as condições atmosféricas em superfície, já que nenhum sistema sinótico atuava na região (Fig. 5b). Assim, a evolução temporal indicada na Fig. 4 é considerada como representativa para o período analisado aqui e será utilizado para inferir a condição atmosférica quanto a estabilidade no momento da medida. Como os perfis da Fig. 3 foram levantados próximo às 10:00 h, será focado a discussão em torno da Fig. 4 entre o início da madrugada e às 10:00 $\mathrm{h}$ do dia 21/09/2016. Como as estimativas do RiB mostraram-se numericamente mais estáveis do que o $\mathrm{Ri}_{\mathrm{G}}$, as discussões em torno da Fig. 4b ficaram centradas nas estimativas do Richardson Bulk.
Devido as grandes diferenças de capacidade térmica da água e do ar, observa-se grandes diferenças entre a temperatura do ar $\left(\mathrm{T}_{\mathrm{ar}}\right)$ e da água ( $\left.\mathrm{T}_{\text {água }}\right)$ a partir de 21:00 h com máxima diferente ocorrendo aproximadamente às $05: 30 \mathrm{~h}$ (Fig. $4 \mathrm{c}$ ), quando a temperatura do ar está ao redor de $\mathrm{T}_{\mathrm{ar}}=15{ }^{\circ} \mathrm{C}$ e a da água $\mathrm{T}_{\text {agua }}=25^{\circ} \mathrm{C}$. Neste período ocorre também as menores diferenças de temperatura entre as alturas 10 e $80 \mathrm{~m}$. Este período é caracterizado por condições fortemente instáveis refletidas nos valores de $\mathrm{RiB}<-0,2$ (Fig. 4d). A partir daí até aproximadamente as 09:00 h, a temperatura a $80 \mathrm{~m}$ fica maior do que a $10 \mathrm{~m}$, tendência esta que inverte a partir das 10:00 $\mathrm{h}$, quando a temperatura a $10 \mathrm{~m}$ fica maior do que a $80 \mathrm{~m}$. A partir do nascer do Sol $(\sim 06: 00 \mathrm{~h})$ inicia-se um processo de aquecimento da $T_{a r}$ que, aproximadamente às 10:00 $\mathrm{h}$ se iguala à $\mathrm{T}_{\text {água }} \mathrm{o}$ que gera uma condição de atmosfera neutra (Fig. 4d). Assim, o fato do perfil do vento a partir do VANT ter sido realizado por volta das 10:00 h, que corresponde a condições de atmosfera neutra, deve explicar o surpreendente ajuste entre o valor de $l$ estimado pelo modelo de Pellegrini e Bodstein (2005) e o observado a partir do VANT.

\section{Conclusões}

Este estudo fez uso, pela primeira vez no Brasil, de um VANT equipado com anemômetro, para medir, diretamente, o perfil vertical do vento influenciado por colinas. Também é inédito no Brasil o uso da morfometria real da colina em estudo, construído a partir dos dados de modelo digital de terreno, como entrada para os modelos. $\mathrm{O}$ aumento relativo da velocidade no topo da colina mostrou
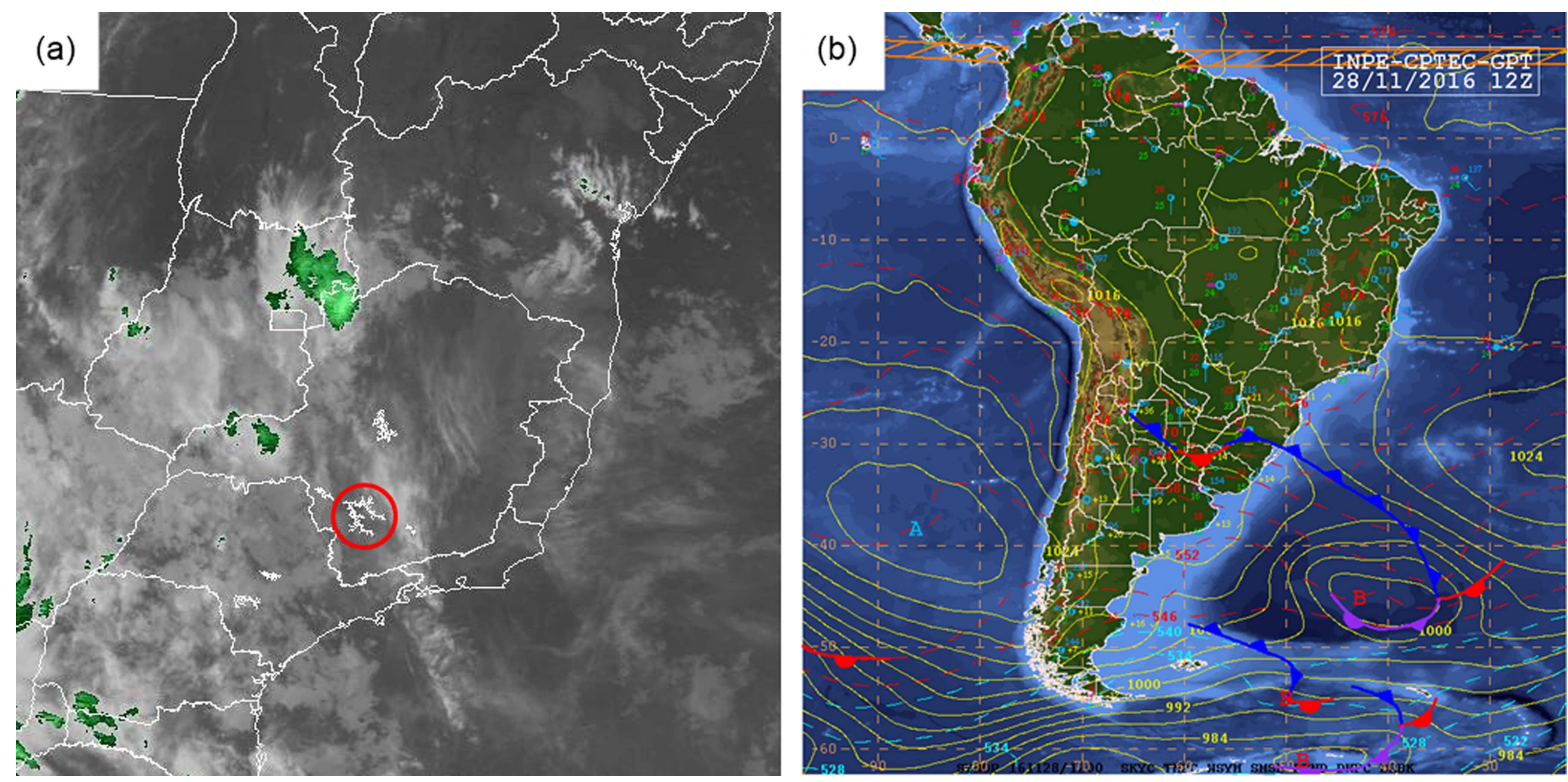

Figura 5 - Imagem IR GOES-13 da região de estudo delimitada pelo círculo (a) e carta sinótica de superfície para o horário mais próximo do experimento. 
ser função de sua altura, o que confirma a teoria. Este valor atingiu $90 \%$ da aceleração a $20 \mathrm{~m}$ de altura, a partir do topo da colina. Embora o experimento tenha sido realizado sob condições ótimas: vento ortogonal ao eixo principal da colina e em condições de estabilidade neutra, a comparação dos valores medidos com os previstos por um modelo analítico amplamente aplicado em colinas, indicou uma surpreendente concordância entre os valores medidos e modelados. Porém, ressalta-se a necessidade de maior coletânea de perfis, com diferentes direções e sob diferentes condições atmosféricas, para um teste mais conclusivo desta teoria. Embora a amostragem necessite ser ampliada e estudos sobre o perfil vertical da turbulência ainda precisam ser realizados, os resultados indicam que a colina em estudo pode ser um local promissor para a instalação de aerogeradores, e que estes poderiam ter alturas próximas a $20 \mathrm{~m}$, o que reduz sensivelmente os custos e dificuldades logísticas. O uso do VANT para o mapeamento do escoamento ao redor de colinas é muito promissor, especialmente pelo fato de que a tecnologia LIDAR de varredura cônica possui limitações de uso em terrenos com topografia irregular. Os resultados apresentados aqui podem fornecer subsídios para outros estudos como dispersão de poluentes em plantas industriais instaladas em colinas, propagação de incêndios nestes terrenos e dispersão de agrotóxicos lançados a partir de avião.

\section{Agradecimentos}

À FAPEMIG (Projeto APQ 1575/14). ATA e FMP agradecem o apoio financeiro do $\mathrm{CNPq}$, respectivamente, pelos projetos CNPq (309315/2015-8) e CNPq (406801/2013-4, 465672/2014-0, 311930/2016-6). À Equinócio Geoambiental pelo fornecimento e suporte ao uso do VANT. Os autores agradecem aos dois revisores pelas importantes sugestões e correções.

\section{Referências Bibliográficas}

ARGAÍN, J.L.; MIRANDA, P.M.A.; TEIXEIRA, MIGUEL A. C. Estimation of the friction velocity in stably stratified boundary-layer flows over hills. Boundary-Layer Meteorology, v. 130, n. 1, p. 15-28, 2009.

ASSIREU, A.T.; MEDONÇA, J.C.; PIMENTA, F.M. Uso de VANT para prospecção eólica em sistemas aquáticos: desenho amostral e avanços instrumentais. (Aceito para publicação na Revista Brasileira de Meteorologia), 2018.

ASSIREU, A.T.; PIMENTA, F.; SOUZA, V. Assessment of the wind power potential of hydroelectric reservoir. Energy Resources: Development, Distribution, and Exploitation, v. 01, p. 1-30, 2011.

ATHANASSIADOU, M.; CASTRO, I.P. Neutral flow over a series of rough hills: a laboratory experiment. BoudaryLayer Meteorology, v. 101, n. 1, p. 1-30, 2001.
AYOTTE, K.W.; HUGHES, D.E. Observations of boundarylayer wind tunnel flow over isolated ridges of varying steepness and roughness. Boundary-Layer Meteorology, v. 112 n. 3 p. 525-556, 2004.

BECHMANN, A.; SØRENSEN, N.N.; BERG, J.; MANN, J.; RÉTHORÉ, P.E. The Bolund Experiment, Part II: Blind Comparison of Microscale Flow Models. Boundary-Layer Meteorology, v. 141, n. 2, p. 245-271, 2011.

BECHMANN, A.; BERG, J.; COURTNEY, M.; JØRGENSEN, H.; MANN, J.; SØRENSEN, N. The Bolund experiment: overview and background. Risø DTU report, 50 p., 2009

BERG, J.; MANN, J.; BECHMANN, A.; COURTNEY, M.S.; JØRGENSEN, H.E. The Bolund experiment, part I: flow over a steep, three-dimensional hill. Boundary-Layer Meteorology, v. 141, n. 2, p. 219-243, 2011.

BITSUAMLAK, G.T.; STATHOPOULOS, T.; BEDARD, C. Numerical evaluation of wind flow over complex terrain. Journal of Aerospace Engineering, v. 17, n. 4, p. 135145,2004

BROWNLOW, R.; LOWRY, D.; THOMAS, R.M.; FISHER, R. E.; FRANCE, J.L.; CAIN, M.; RICHARDSON, T.S.; GREATWOOD, C.; FREER, J.; PYLE, J.A. MACKENZIE, A.R.; NISBET, E. G. Methane mole fraction and $\delta 13 \mathrm{C}$ above and below the trade wind inversion at Ascension Island in air sampled by aerial robotics. Geophysical Research Letters, v. 43, n. 22, p. 11893-11902, 2016.

CAO, S.; TAMURA T. Experimental study on roughness effects on turbulent boundary layer flow over a two-dimensional steep hill. Journal of Wind Engineering and Industrial Aerodynamics, v. 94, n. 1, p. 1-19, 2006.

CASTRO F.; PALMA, J.M.L.M.; SILVA LOPES, A. Simulation of the askervein flow. Part 1: Reynolds averaged NavierStokes Equations. (k- $\mathrm{k}-\varepsilon$ turbulence model). BoundaryLayer Meteorology, v. 107, n. 3, p. 501-530, 2003.

CHAUDHARI, A.; HELLSTEN, A.; AGAFONOVA, O.; HÄMÄLÄINEN, J. Large eddy simulation of boundarylayer flows over two-dimensional hills. Progress in Industrial Mathematics at ECMI 2012, v. 19, p. 211-218, 2014.

DIAS, N.L.; GONÇALVES, J.E.; FREIRE, L.S.; HAZEGAWA, T.; MALHEIROS, A.L. Obtaining potential virtual temperature profiles, entrainment fluxs, and spectra from mini unmanned aerial vehicle data. Boundary-Layer Meteorology, v. 145, n.1, p. 93-111, 2012.

DI MAURO, B.; FAVA, F.; FERRERO, L.; GARZONIO, R.; BACCOLO, G.; DELMONTE, B.; COLOMBO, R. Mineral dust impact on snow radiative properties in the European Alps combining ground, UAV, and satellite observations. Journal of Geophysical Research: Atmospheres, v. 120, n. 12, p. 6080-6097, 2015.

FINNIGAN, J.J. The logarithmic wind profile in complex terrain. CSIRO Environmental Mechanics Technical Report, v. 14, n. T44, 69 p., 1992.

FORTHOFER, J.M.; BUTLER, B.W.; WAGENBRENNER, N.S. A comparison of three approaches for simulating fine scale surface winds in support of wildland fire management. Part II. An exploratory study of the effect of simulated winds on fire growth simulations. International Journal of Wildland Fire, v. 23, n. 7, p. 982-994, 2014.

HOLMAN, B.P.; LAZARUS, S.M.; SPLITT, M.E.A. FetchBased Statistical Method to Bias Correct and Downscale 
Wind Speed over Unresolved Water Bodies. Weather and Forecasting, v. 32, n. 4, p. 1637-1657, 2017.

HUNT, J.C.R. Wind over hills. In: Workshop on the Planetary Boundary Layer, p. 107-144, Boston, AMS public, 1980.

JOHNSON, W.; KELLEY, N. Design specifications for the development of the initial validation Software (Version 3.0) for processing of NWTC 80-meter meteorological tower data. National Renewable Energy Lab., Golden, CO (US), 2000.

KILPATRICK, R.; HANGAN, H.; SIDDIQUI, K.; PARVU, D.; LANGE, J.; MANN, J.; BERG, J. Effect of Reynolds number and inflow parameters on mean and turbulent flow over complex topography. Wind Energy Science, v. 1, n. 2, p. 237-254, 2016.

LEE, S.J.; LIM, H.C.; PARK, K.C. Wind flow over sinusoidal hilly obstacle located in a uniform flow. Wind and Structures, v. 5, n. 6, p. 515-526, 2002.

LIU, Z.; ISHIHARA, T.; TANAKA, T.; HE, X. LES study of turbulent flow fields over a smooth 3-D hill and a smooth 2D ridge. Journal of Wind Engineering and Industrial Aerodynamics, v. 153, p. 1-12, 2016.

LOPES, A.S.; PALMA, J.M.L.M.; CASTRO, F.A. Simulation of the Askervein flow, Part 2: large eddy simulations. Boundary-Layer Meteorology, v. 125, n. 1, p. 85-108, 2007.

LUBITZ, W.D.; WHITE, B.R. Wind tunnel and field investigation of the effect of local wind direction on speed-up over hills. Journal of Wind Engineering and Industrial Aerodynamics, v. 95, n. 8, p. 639-661, 2007

MANIKANTAN, H.; PRUTHVIRAJ, U. Pollutant dispersion in the wake of a hill: a numerical analysis. Journal of Environmental Research and Development, v. 5, n. 2, p. 330339, 2010.

MATTUELLA, J.M.L.; LOREDO-SOUZA, A.M.; OLIVEIRA, M.G.K.; PETRY, A.P. Wind tunnel experimental analysis of a complex terrain micrositing. Renewable and Sustainable Energy Reviews, v. 54, p. 110-119, 2016.

MEDEIROS, L.E.; MAGNAGO, R.D.O.; FISCH, G.; MARCIOTTO, E.R. Observational study of the surface layer at an ocean-land transition region. Journal of Aerospace Technology and Management, v. 5, n. 4, p. 449-458, 2013

NEWMAN, J.F.; KLEIN, P.M. The impacts of atmospheric stability on the accuracy of wind speed extrapolation methods. Resources, v. 3, n. 1, p. 81-105, 2014.

NIEMINSKI, N.M.; GRAHAM, S.A. Modeling Stratigraphic Architecture Using Small Unmanned Aerial Vehicles and Photogrammetry: Examples From the Miocene East Coast Basin, New Zealand. Journal of Sedimentary Research, v. 87, n. 2, p. 126-132, 2017.

ORE, J.P.; ELBAUM, S.; BURGIN, A.; DETWEILER, C. Autonomous aerial water sampling. Journal of Field Robotics, v. 32, n. 8, p. 1095-1113, 2015.

PAIVA, L.M.S.; BODSTEIN, G.C.R.; MENEZES, W.F.; Simulação do escoamento atmosférico sobre uma colina isolada utilizando o modelo RAMS. Revista Brasileira de Meteorologia, v. 19, n. 2, p. 163-176, 2004.

PALOMAKI, R.T., NATHAN, T.R., BOSSHE, M.V.D., SHERMAN, T.J., WEKKER, S.F.J. Wind estimation in the lower atmospheric using multirotor aircraft. Journal of
Atmospheric and Oceanic Technology, v. 34, n. 5, p. 1183-1191, 2017.

PELLEGRINI, C.C.; BODSTEIN, G.C.R. On the Height of Maximum Speed-up in Atmospheric Boundary layers over low hills. In: 1st National Congress of Mechanical Engineering (I CONEM), 2000.

PELLEGRINI, C.C.; BODSTEIN, G.C.R. A modified logarithmic law for neutrally stratified flow over low-sloped hills. Journal of Applied Meteorology, v. 44, n. 6, p. 900-916, 2005.

POGGI, D.; KATUL, G.G.; FINNIGAN, J.J.; BELCHER, S.E. Analytical models for the mean flow inside dense canopies on gentle hilly terrain. Quarterly Journal of the Royal Meteorological Society, v. 134, n. 634, p. 1095-1112, 2008.

RIPPIN, D.M.; POMFRET, A.; KING, N. High resolution mapping of supra-glacial drainage pathways reveals link between micro-channel drainage density, surface roughness and surface reflectance. Earth Surface Processes and Landforms, v. 40, n. 10, p. 1279-1290, 2015.

SALMON, J.R.; BOWEN, A. J.; HOFF, A.M.; JOHNSON, R.; MICKLE, R.E.; TAYLOR, P.A.; TETZLAFF, G.; WALMSLEY, J. L. The Askervein Hill experiment: Mean wind variations at fixed heights above the ground. Boundary-Layer Meteorology, v. 43, n. 3, p. 247-271, 1988a.

SALMON, J.R., TEUNISSEN, H.W., MICKLE, R.E.; TAYLOR, P. A. The Kettles Hill Project: Field Observations, WindTunnel Simulations and Numerical Model Predictions for Flow over a Low Hill. Boundary-Layer Meteorology. v. 43, n. 3, p. 309-343, 1988 b.

SCHMIDT, J.; STOEVESANDT, B. Modelling complex terrain effects for wind farm layout optimization. In: Journal of Physics: Conference Series, v. 524, n. 1, p. 1-10, 2014.

SHIMADA, S.; OHSAWA, T.; OHGISHI, T.; KIKUSHIMA, Y.; KOGAKI, T.; KAWAGUCHI, K.; NAKAMURA, S. Offshore wind profile measurements using a Doppler LIDAR at the Hazaki Oceanographical Research Station. In: International Conference on Optical Particle Characterization (OPC 2014). International Society for Optics and Photonics. v. 9232, p. 92320R - 92320R-6, 2014.

SPENCE, C.; MENGISTU, S. Deployment of an unmanned aerial system to assist in mapping an intermittent stream. Hydrological Processes, v. 30, n. 3, p. 493-500, 2016.

STULL, R.B. An Introduction to Boundary Layer Meteorology. Kluwer Academic Publishers, 177 p., 1991.

TAYLOR, P.A.; LEE, R.J. Simple guide lines for estimating wind speed variations due to small scale topographic features. Climatol. Bull, v. 18, n. 2, p. 3-32, 1984.

VERBURG, P.; ANTENUCCI J.P. Persistent unstable atmospheric boundary layer enhances sensible and latent heat loss in a tropical great lake: Lake Tanganyika. Journal of Geophysical Research. v. 115, n. D11109, p. 1-13, 2010.

VIVONI, E. R.; RANGO, A.; ANDERSON, C.A.; PIERINI, N. A.; SCHREINER-MCGRAW, A.P.; SARIPALLI, S.; LALIBERTE, A.S. Ecohydrology with unmanned aerial vehicles. Ecosphere, v. 5, n. 10, p. 1-14, 2014.

VLADUT, A.C., COSOIU, C.I., GEORGESCU, A.M., DEGERATU, M., DAMIAN, R.M. Wind tunnel and numerical 
modeling of atmospheric boundary layer flow over Bolund Island. Energy Procedia, v. 85, p. 603-611, 2016.

WALMSLEY. J.L.; TAYLOR, P.A. Boundary layer flow over topography: Impacts of the Askervein study. BoundaryLayer Meteorology, v. 78, n.3, p. 291-320, 1996.
WOOD, N. The onset of flow separation in neutral, turbulent flow over hills. Boundary-Layer Meteorology, v. 76, n. 12, p. 137-164, 1995.

This is an Open Access article distributed under the terms of the Creative Commons Attribution License, which permits unrestricted use, distribution, and reproduction in any medium, provided the original work is properly cited. 